\title{
Anticyclonic precession of a plume in a rotating environment
}

DOI:

10.1002/2017GL074191

\section{Document Version}

Final published version

Link to publication record in Manchester Research Explorer

\section{Citation for published version (APA):}

Frank, D., Landel, J. R., Dalziel, S. B., \& Linden, P. F. (2017). Anticyclonic precession of a plume in a rotating environment. Geophysical Research Letters, 44(18). https://doi.org/10.1002/2017GL074191

\section{Published in:}

Geophysical Research Letters

\section{Citing this paper}

Please note that where the full-text provided on Manchester Research Explorer is the Author Accepted Manuscript or Proof version this may differ from the final Published version. If citing, it is advised that you check and use the publisher's definitive version.

\section{General rights}

Copyright and moral rights for the publications made accessible in the Research Explorer are retained by the authors and/or other copyright owners and it is a condition of accessing publications that users recognise and abide by the legal requirements associated with these rights.

\section{Takedown policy}

If you believe that this document breaches copyright please refer to the University of Manchester's Takedown Procedures [http://man.ac.uk/04Y6Bo] or contact uml.scholarlycommunications@manchester.ac.uk providing relevant details, so we can investigate your claim.

\section{OPEN ACCESS}




\section{RESEARCH LETTER}

10.1002/2017GL074191

\begin{abstract}
Key Points:
-We report a new phenomenon in

the dynamics of plumes in a rotating environment: The anticyclonic precession of the plume axis

- The precession is independent of the source size, the buoyancy flux, and the water depth and scales linearly with the rotation rate

- We infer that the plume in a rotating environment will always start to precess if it is maintained over a sufficiently long time
\end{abstract}

Supporting Information:

- Supporting Information S1

- Text S1

- Figure S1

- Table S1

- Movie S1

Correspondence to:

D. Frank,

D.Frank@damtp.cam.ac.uk

\section{Citation:}

Frank, D., J. R. Landel, S. B. Dalziel, and P. F. Linden (2017), Anticyclonic precession of a plume in a rotating environment, Geophys. Res. Lett., 44, 9400-9407, doi:10.1002/2017GL074191.

Received 19 MAY 2017 Accepted 14 AUG 2017 Accepted article online 16 AUG 2017 Published online 18 SEP 2017

(2)17. American Geophysical Union. All Rights Reserved.

\section{Anticyclonic precession of a plume in a rotating environment}

\author{
D. Frank ${ }^{1} \mathbb{D}^{\mathbb{D}}$, J. R. Landel ${ }^{2} \mathbb{D}$, S. B. Dalziel ${ }^{1} \mathbb{D}$, and P. F. Linden ${ }^{1}$ \\ ${ }^{1}$ Department of Applied Mathematics and Theoretical Physics, University of Cambridge, Cambridge, UK, ${ }^{2}$ School of \\ Mathematics, University of Manchester, Manchester, UK
}

\begin{abstract}
Motivated by potential effects of the Earth's rotation on the Deepwater Horizon oil plume, we conducted laboratory experiments on saltwater point plumes in a homogeneous rotating environment across a wide range of Rossby numbers $0.02 \leq R o \leq 1.3$. We report a striking physical instability in the plume dynamics near the source: after approximately one rotation period, the plume tilts laterally and starts to precess anticyclonically. The mean precession frequency $\bar{\omega}$ scales linearly with the rotation rate $\Omega$ as $\bar{\omega} \approx 0.4 \Omega$. We find no evidence of a critical Rossby number above which precession ceases. We infer that a conventionally defined Rossby number is not an appropriate parameter when the plume is maintained over a long time: provided $\Omega \neq 0$, rotation is always important to the dynamics. This indicates that precession may occur in persistent oceanic or atmospheric plumes even at low latitudes.
\end{abstract}

\section{Introduction}

The unprecedented conditions of the 2010 Deepwater Horizon (DwH) oil plume revealed features of plume dynamics that are yet to be fully understood. A hot mixture of oil, gas, water, and sediment was discharged into a cold saltwater environment at $1500 \mathrm{~m}$ depth. Socolofsky et al. [2011] estimated the source buoyancy flux between $B_{0}=0.48$ and $0.98 \mathrm{~m}^{4} \mathrm{~s}^{-3}$. Currents, ocean stratification, chemical processes, and pressure changes affected the plume during its rise. Furthermore, the oil plume evolved in a rotating environment. The Rossby number is conventionally $R o=\left(B_{0} \Omega^{-3}\right)^{1 / 4} H^{-1}$ in a nonstratified environment (with $\Omega$ the rotation rate and $H$ the water depth), and $R o_{s}=N / f$ in a stratified environment (with $N$ the buoyancy frequency and $f$ the Coriolis parameter). The expectation for $R o \approx 3-20$ [Socolofsky et al., 2011] is a plume insensitive to rotation. However, these definitions of Ro only account for rotation effects during the initial plume rise. Since the DwH plume persisted for several months, entraining fluid from increasing distances, it is possible that it was influenced by the Earth's rotation.

Experiments on saltwater plumes in a homogeneous rotating environment were conducted by Fernando et al. [1998] and Goodman et al. [2004], and in a stratified rotating environment by Helfrich and Battisti [1991] at $R o>0.3,0.03<R o<0.13$ and $0.67<R o_{s}<4.28$, respectively. These studies show that a cyclonic vortex develops around the plume, and the plume changes from a conical to a cylindrical shape because the rotation inhibits entrainment. After reaching a solid boundary or a neutral density level, the plume spreads laterally until its front becomes baroclinically unstable. Fernando et al. [1998] determined the initial time $t_{c}=2.4 / \Omega$ when the plume feels the background rotation, i.e., when its vertical descent is slowed compared to the nonrotating case. They report that plumes with $R o>0.3$ are only impacted by rotation after impinging on the tank boundary. None of these experimental studies [Helfrich and Battisti, 1991; Fernando et al., 1998; Bush and Woods, 1999; Goodman et al., 2004; Yamamoto et al., 2011] reported observing plume precession. In their turbulence-resolving simulations at Ro comparable with the DwH plume, Fabregat Tomàs et al. [2016] reported lateral deflection of the plume and onset of anticyclonic precession.

Motivated by possible effects of the Earth's rotation on the $\mathrm{DwH}$ plume and geophysical plumes in general, we examine the influence of background rotation on the plume through laboratory experiments at $0.02 \leq R o \leq 1.3$. This range includes plumes affected by rotation both before and after impinging on the tank boundary. Our main result is that we have observed a new phenomenon in the plume dynamics that, to the best of our knowledge, has not been previously reported in experimental studies: near the source, the plume is laterally displaced off the centerline and precesses in the anticyclonic direction with a relatively well-defined precession frequency $\bar{\omega}$. This observation appears to be independent of the Rossby number of 


Table 1. Summary of the Experimental Parameters
\begin{tabular}{lccccccc} 
a \\
Series & Tank & $H(\mathrm{~cm})$ & $d_{0}(\mathrm{~cm})$ & $B_{0}\left(\mathrm{~cm}^{4} \mathrm{~s}^{-3}\right)$ & $\Omega\left(\mathrm{rad} \mathrm{s}^{-1}\right)$ & $\Gamma$ & $R o$ \\
\hline$A$ & $\mathrm{~B}$ & 110 & 0.75 & $\approx 23$ & $0.1-0.8$ & $0.9-1.0$ & $0.02-0.1$ \\
$B$ & $\mathrm{~B}$ & 110 & 0.75 & $\approx 300$ & $0.1-0.8$ & $0.7-0.8$ & $0.04-0.2$ \\
C & $\mathrm{B}$ & 110 & 0.3 & $\approx 36$ & $0.1-0.8$ & 0.35 & $0.04-0.12$ \\
$D$ & $\mathrm{~B}$ & 30 & 0.75 & $\approx 23$ & $0.1-0.8$ & $1-1.1$ & $0.08-0.4$ \\
E & $\mathrm{B}$ & 20 & 0.75 & $\approx 23$ & $0.1-0.8$ & $1-1.1$ & $0.12-0.6$ \\
$F$ & $\mathrm{~B}$ & 10 & 0.75 & $\approx 23$ & $0.1-0.8$ & $0.9-1.1$ & $0.25-1.3$ \\
$G$ & $\mathrm{~A}$ & 40 & 0.75 & $\approx 23$ & $0.2-1$ & $0.9-1.2$ & $0.05-0.2$ \\
$H$ & $\mathrm{~A}$ & 40 & 1 & $\approx 6$ & $0.2-1$ & $0.1-0.5$ & $0.07-0.3$ \\
I & $\mathrm{A}$ & 40 & 0.3 & $\approx 90$ & $0.2-1$ & $1.15-1.2$ & $0.04-0.14$ \\
\hline
\end{tabular}

${ }^{\mathrm{a}}$ Additional details for each series are presented in Table $\mathrm{S} 1$ in the supporting information.

the plume. After detailing the phenomenology of the anticyclonic plume precession, we present a semiempirical model that agrees quantitatively with the observations.

\section{Experiments}

We conducted over 100 experiments, varying $H, \Omega$, nozzle diameter $d_{0}$, and $B_{0}=\pi g^{\prime} u_{0} d_{0}^{2} / 4$, with $u_{0}$ the source velocity, $g^{\prime}=g\left(\rho_{0}-\rho_{a}\right) / \rho_{a}$ the source reduced gravity, $g$ the gravity constant, $\rho_{0}$ the source density, and $\rho_{a}$ the environment density (see Table 1 and further details in the supporting information). Two different tanks were used for the experiments (Figure 1): a small tank A with a circular base of diameter $74 \mathrm{~cm}$ and height $50 \mathrm{~cm}$, and a large tank $B$ with an octagonal base of maximum width $100 \mathrm{~cm}$ and height $120 \mathrm{~cm}$. The plume was produced by discharging salt water from a constant-head tank through a nozzle positioned a few centimeters below the water surface. The plumes were started after the water in the tank was in a solid-body rotation. The flow was recorded using two JAI CVM4+MCL 1.3 megapixel cameras attached to the turntable to capture side and top views (Figure 1; see supporting information for more details). We produced "pure" plumes by ensuring that the Richardson number $\Gamma=g^{\prime} d_{0} /\left(2 \alpha u_{0}^{2}\right) \approx 1$, with the entrainment constant $\alpha \approx 0.1$. The source momentum could be neglected as the jet length $L_{j}=2-4 \mathrm{~cm} \ll H$.

\section{Results}

\subsection{Qualitative Flow Observations}

Figure 2 presents time series of side view snapshots showing the plume evolution at $R o \approx 0.18,0.12$, and 0.09 (from left to right), with $\Omega=0.3,0.5$, and $0.8 \mathrm{rad} \mathrm{s}^{-1}$, respectively, and $H=30 \mathrm{~cm}, d_{0}=0.75 \mathrm{~cm}, B_{0} \approx 23 \mathrm{~cm}^{4} \mathrm{~s}^{-3}$. Similar to Fernando et al. [1998], we observed that the initial descent of the plume was unaffected by background rotation (see Figure 2, first row, for $t=0.2 T$, with $T=2 \pi / \Omega$ the rotation period), and then a cyclonic circulation formed in the ambient fluid (see Movie S1 in the supporting information), before a cylindrical column of reentrained dyed fluid gradually developed around the plume. After $t \approx 0.5 T$ to $T$, the plume was deflected laterally and discharged at an angle to the vertical (see Figure 2 for $t \geq T$ ). The deflection onset is to be contrasted with the critical time $t_{c} \approx 2.4 / \Omega \approx 0.38 T$ determined by Fernando et al. [1998] for which the background rotation starts to affect the plume dynamics.

Once the plume was deflected laterally, it precessed anticyclonically with a well-defined precession frequency $\bar{\omega}$ increasing with $\Omega$. Moreover, the region of the visible plume precession in which we can clearly recognize the structure of the downward discharging plume is spatially limited (Figure 2). Further downstream, the coherence of the plume structure is lost and the downward directed plume disintegrates in (or is obscured by) a cloud of dyed fluid. The vertical extent of the precession region seems to decrease with increasing $\Omega$ (arrows in Figure 2) and decreasing $B_{0}$ (not shown here). In Figure 3 (top), we plot the vertical extent $h$ of the precession region for experiments with $B_{0} \approx 6 \mathrm{~cm}^{4} \mathrm{~s}^{-3}, 23 \mathrm{~cm}^{4} \mathrm{~s}^{-3}$, and $300 \mathrm{~cm}^{4} \mathrm{~s}^{-3}$. For each experiment, we selected 10 images (one every 30 to $40 \mathrm{~s}$ ) and determined $h$ by visually tracking the plume from the nozzle until we could no longer recognize a coherent plume structure. Figure 3 (top) shows the mean $h$ for each experiment, with error bars indicating the uncertainty. Overall, $h$ scales approximately linearly with the length scale $L_{B \Omega}=\left(B_{0} / \Omega^{3}\right)^{1 / 4}$ and does not depend on the nozzle diameter $d_{0}$. For $L_{B \Omega} \lesssim 2 \mathrm{~cm}$, the cloud of dyed fluid almost completely obscured the nozzle and made it difficult to quantify $h$ or the precession frequency. 

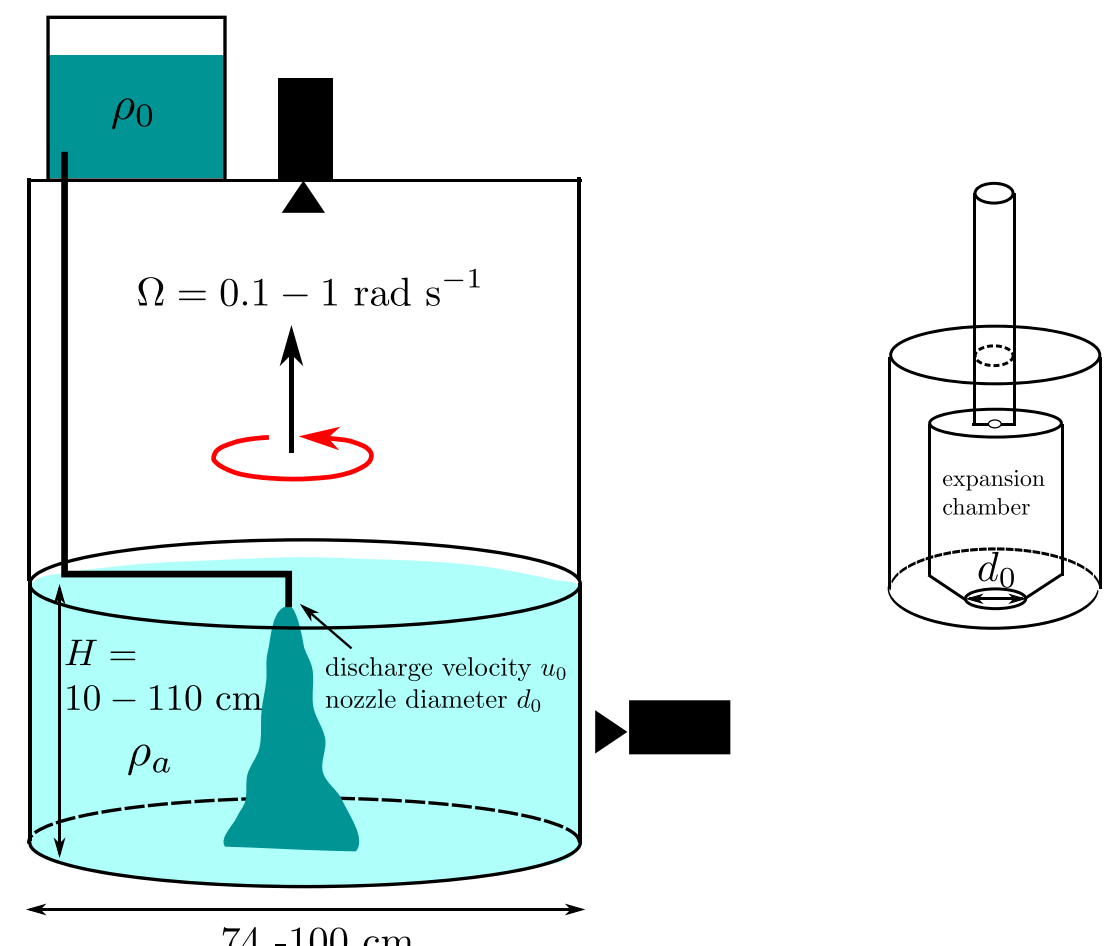

$74-100 \mathrm{~cm}$

Figure 1. (left) Schematic of the experimental setup: a turbulent saltwater plume was discharged vertically downward in a rotating freshwater homogeneous environment, initially in solid-body rotation. A top view and a side view camera recorded the plume dynamics, rendered visible by blue dye. The Rossby number was varied between $R o=\left(B_{0} \Omega^{-3}\right)^{1 / 4} H^{-1}=0.02$ and 1.3 by changing the source buoyancy flux $B_{0}$, the rotation rate $\Omega$, and the water depth $H$. (right) Sketch of the inner structure of the plume nozzle, designed to ensure the plume was turbulent on exiting the nozzle.

The average deflection angle of the plume from the vertical appeared to be constant $\bar{\psi} \approx 30^{\circ} \pm 11^{\circ}$ across all experiments, with a relatively large error indicating that the instantaneous deflection angle $\psi$ varied a lot within the same experiment.

\subsection{Plume Precession}

To extract the plume precession frequency, we first determined the lateral location of the plume centerline, as a function of time $t$ and distance $z$ from the source in the visible precession region, using an algorithm described in the supporting information. Figure 3 (middle) presents a typical time series of the measured centerline position at $z=5 d_{0}$ from the nozzle (thick blue curve), which clearly shows that the plume performs a periodic motion. The time series were then analyzed using the Hilbert-Huang transform [Huang and Attoh-Okine, 2005]. We first decomposed the signal into a set of intrinsic mode functions (IMFs) using an empirical mode decomposition (see Figure 3, middle). The most energetic IMF (IMF7) contains information about the precession frequency $\omega(t)$, retrieved by applying the Hilbert transform. As $\omega$ can be a function of time, the average precession frequency $\bar{\omega}$ was determined by taking the median value of $\omega(t)$ (to reduce the effects of unphysical outliers). In Figure 3 (bottom), we plot $\bar{\omega}$ as a function of $\Omega$ for all our experiments. The precession frequency $\bar{\omega}$ is independent of $d_{0}, B_{0}, H$, and the geometry of the domain. Furthermore, $\bar{\omega}$ scales linearly with the rotation rate, such that

$$
\bar{\omega} \approx(0.4 \pm 0.04) \Omega
$$

\subsection{Spinning Top Model for the Plume Precession}

We propose a model for the plume precession inspired by spinning tops. As Fernando et al. [1998] showed, a rotating plume forms a cylindrical column of fluid of a different density from the environment. Owing to the adverse pressure gradient encountered in a rotating environment, in accordance with the Taylor-Proudman theorem and as shown numerically by Fabregat Tomàs et al. [2016], the plume is deflected from the vertical. Then, in the precession region, it forms a cylindrical column of height $h_{c}=C_{h}\left(B_{0} \Omega^{-3}\right)^{1 / 4}$ and radius $r_{c}=$ $C_{r}\left(B_{0} \Omega^{-3}\right)^{1 / 4}$, from dimensional analysis, with some constants $C_{h}$ and $C_{r}$ (Figure 4, left). 


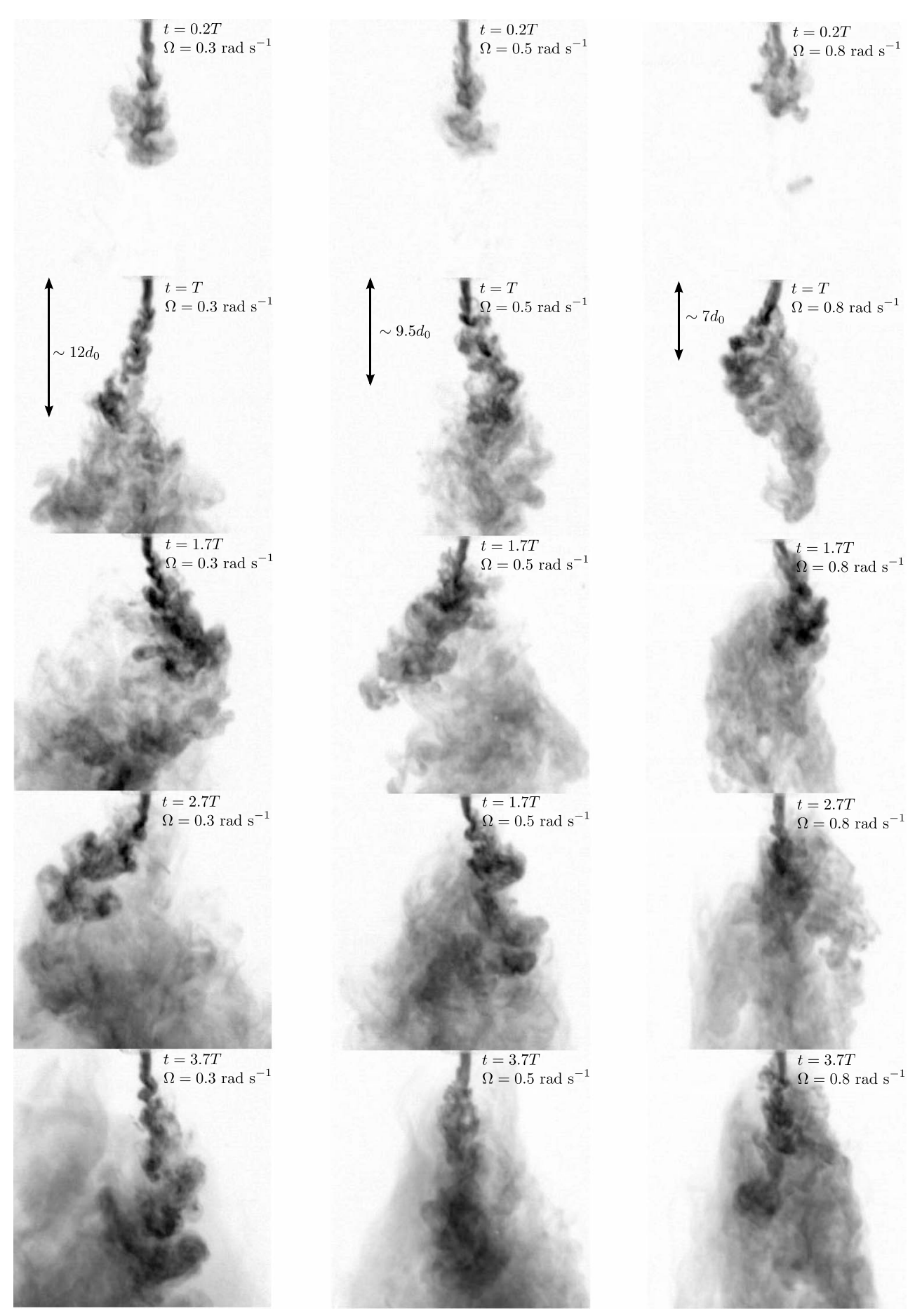

Figure 2. Time series of greyscale pictures showing the plume at times $t=0.2 T, t=T, t=1.7 T, t=2.7 T$, and $t=3.7 T$ (with $T=2 \pi / \Omega$ the rotation period) after the start of the plume for $d_{0}=0.75 \mathrm{~cm}, B_{0} \approx 23 \mathrm{~cm}^{4} \mathrm{~s}^{-3}, H=30 \mathrm{~cm}$ (Series $D$ ), and for (left column) $\Omega=0.3 \mathrm{rad} \mathrm{s}^{-1}$, (middle column) $\Omega=0.5 \mathrm{rad} \mathrm{s}^{-1}$, and (right column) $\Omega=0.8 \mathrm{rad} \mathrm{s}^{-1}$. The corresponding Rossby numbers are $R o \approx 0.18,0.12$, and 0.09 , respectively. The lateral deflection of the plume away from the vertical axis and its precession around the vertical axis are clearly visible after $t=T$. We find the precession frequency to depend linearly on $\Omega$ but independent of $R o$. 

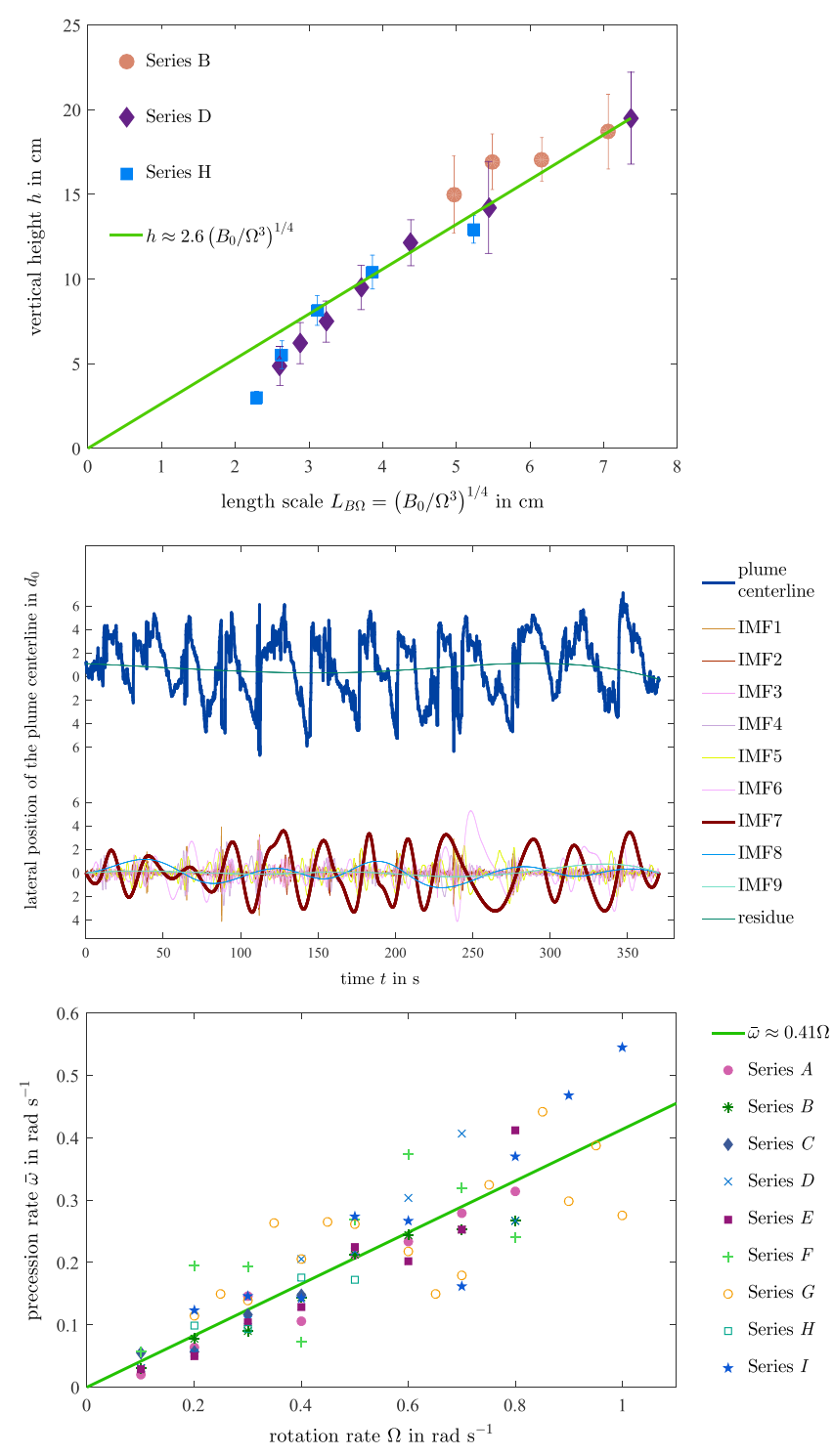

Figure 3. (top) Vertical extent $h$ of the visible precession region of the plume, as a function of the buoyancy length scale $L_{B \Omega}$. (middle) Typical time series of the position of the plume centerline determined from the side view recordings at the distance $z=5 d_{0}$ from the nozzle, $d_{0}=0.75 \mathrm{~cm}, B_{0} \approx 22 \mathrm{~cm}^{4} \mathrm{~s}^{-3}, H=40 \mathrm{~cm}$, and $\Omega=0.5 \mathrm{rad} \mathrm{s}^{-1}$. The signal is decomposed into nine intrinsic mode functions (IMFs) using the Hilbert-Huang transform [Huang and Attoh-Okine, 2005]. (bottom) Mean plume precession frequency $\bar{\omega}$ as a function of the rotation rate of the environment $\Omega$. The parameters for the experimental series are listed in Table 1.

In the rotating frame of reference (R-FoR), the plume rotates cyclonically with angular velocity $\omega_{r}$, assumed to be the same as the velocity of the rim current whose existence was shown by Fernando et al. [1998]. In the laboratory frame of reference $(\mathrm{L}-\mathrm{FoR})$, the plume rotates with angular velocity $\Omega+\omega_{r}$. The angular momentum of the plume is $L=I \omega_{r}$ (R-FoR) or $L=I\left(\Omega+\omega_{r}\right)$ (L-FoR), with $/$ the moment of inertia. Due to the adverse pressure gradient, there is a force $\boldsymbol{F}_{p}$ opposed to the plume, of similar magnitude as the gravitational force $\boldsymbol{F}_{g}$ acting on the center of mass of the cylinder (see Figure 4, left). Fabregat Tomàs et al. [2016] showed that, in a rotating environment, the adverse pressure gradient balances approximately the buoyancy force of the plume. Assuming that $\boldsymbol{F}_{p}$ and $\boldsymbol{F}_{g}$ are not collocated, the resulting torque induces a cyclonic precession in L-FoR. Invoking the formula for the precession of a spinning top, the precession frequency $\omega_{p}$ in L-FoR is

$$
\omega_{p}=\frac{m_{c} g_{c}^{\prime} \ell}{I\left(\omega_{r}+\Omega\right)}=\frac{g_{c}^{\prime} \ell}{r_{c}^{2}\left(\omega_{r}+\Omega\right)},
$$



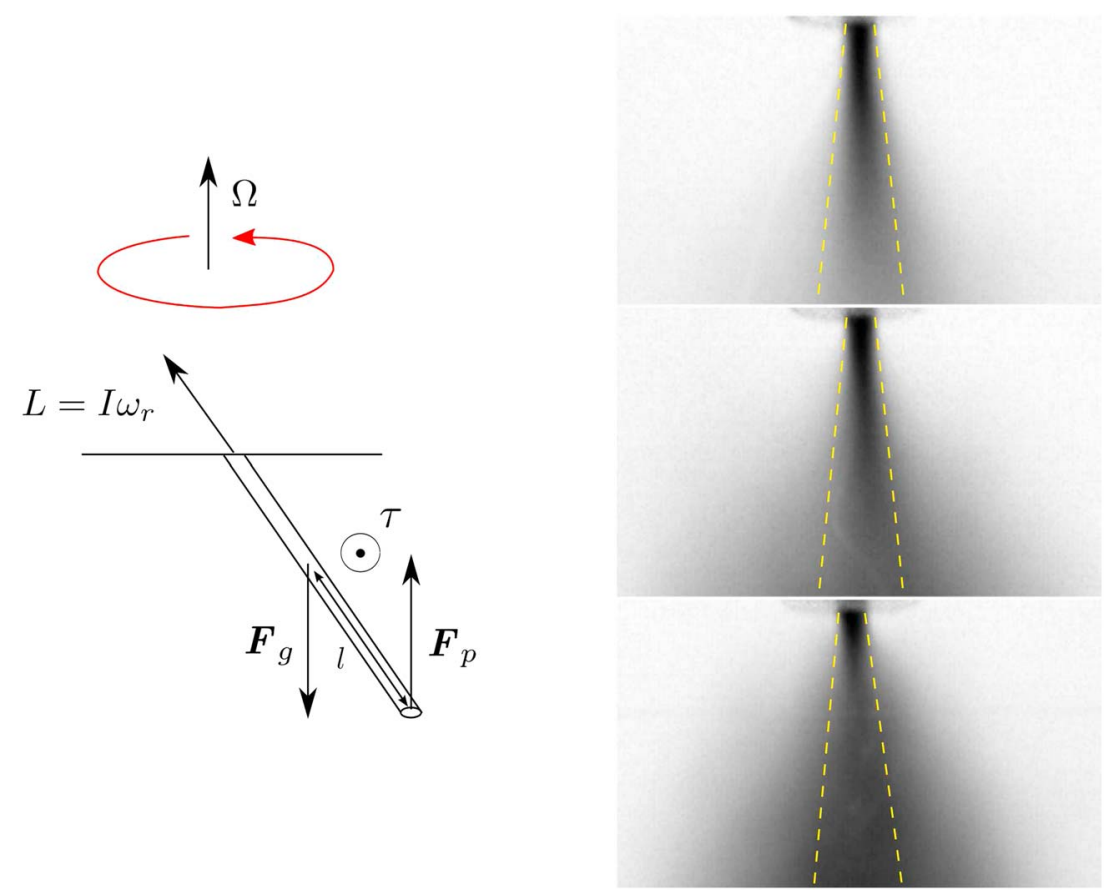

Figure 4. (left) Schematic side view of a laterally deflected plume modeled as a columnar spinning top. The plume rotates at rotation speed $\omega_{r}$ due to the rim current [Fernando et al., 1998] and is subject to buoyancy force $\boldsymbol{F}_{g}$ and adverse pressure gradient $\boldsymbol{F}_{p}$. As these two forces are not collocated and separated with distance $\ell$, the resulting torque $\tau$ induces plume precession in a rotating environment with background rotation rate $\Omega$. (right) Averaged dye distribution for the first $3 \mathrm{~min}$ of the experiments for $H=30 \mathrm{~cm}, B_{0} \approx 23 \mathrm{~cm}^{4} \mathrm{~s}^{-3}, d_{0}=0.75 \mathrm{~cm}$, and (top right) $\Omega=0.1 \mathrm{rad} \mathrm{s}^{-1}$ (middle right) $\Omega=0.5 \mathrm{rad} \mathrm{s}^{-1}$, and (bottom right) $\Omega=0.7 \mathrm{rad} \mathrm{s}^{-1}$. The vertical extent of the region shown is $10 d_{0}$. The dashed yellow lines indicate the theoretical shape of a plume in a nonrotating environment for the entrainment constant $\alpha=0.1$. Clearly, the dye dispersion in a rotating environment is increased compared to a nonrotating environment.

with $\ell$ the distance between $\boldsymbol{F}_{p}$ and $\boldsymbol{F}_{g^{\prime}} g_{c}^{\prime}$ the reduced gravity acceleration of the cylinder, $m_{c}$ the mass of the cylinder, and $I=m_{c} r_{c}^{2}$ its moment of inertia. We expect that $\ell=C_{f} h_{c}$, with $C_{f}$ a constant.

As experimentally observed, the precession starts after $t \approx 2 \pi / \Omega$. Thus, we have

$$
g_{c}^{\prime} \approx \frac{2 \pi B_{0}}{\Omega \pi h_{c} r_{c}^{2}}=\frac{2 B_{0}}{\Omega r_{c}^{2} h_{c}} .
$$

The strength of the rim current (and thus the rotation rate of the cylindrical column) is $\omega_{r} \approx 2 \Omega \Delta r / r_{c}=$ $2 \Omega \beta h_{c} / r_{c}$, where $\Delta r$ is the horizontal displacement of the ambient fluid due to turbulent entrainment into the plume. Since the entrainment velocity is proportional to the plume centerline velocity, $\Delta r \approx \beta h_{c}$ with some proportionality factor $\beta$ [Fernando et al., 1998]. Substituting $\ell, g_{c^{\prime}}^{\prime}$ and $\omega_{r}$ in (2) yields

$$
\omega_{p}=\frac{2 C_{f} \Omega}{C_{r}^{4}\left(2 \beta C_{h} / C_{r}+1\right)},
$$

confirming our observation that the precession rate is proportional to $\Omega$. Equation (4) is an estimate for the precession frequency of the plume in L-FoR, in which it precesses in the cyclonic direction. To obtain the precession frequency (and direction) in R-FoR, we consider the angular velocity of the environment and the cyclonic rim current $\left(\sim 2 \Omega \beta h_{c} / r_{c}\right)$, which also deflects the plume in the cyclonic direction. Thus, in R-FoR, we obtain

$$
\bar{\omega}=\frac{2 C_{f} \Omega}{C_{r}^{4}\left(2 \beta C_{h} / C_{r}+1\right)}-\Omega+2 \Omega \beta C_{h} / C_{r} .
$$

Fernando et al. [1998] determined that the plume starts to feel the presence of background rotation when $h_{c} \approx 3.3\left(B_{0} \Omega^{-3}\right)^{1 / 4}$ and the maximum width is $r_{c} \approx 1.4\left(B_{0} \Omega^{-3}\right)^{1 / 4}$, giving $C_{h} \approx 3.3$ and $C_{r} \approx 1.4$. We assume 
that the distance between the two forces is half the cylinder height, hence $C_{f} \approx 1 / 2$. It is also reasonable to assume that $\beta \approx \alpha \approx 0.1$, yielding

$$
\bar{\omega} \approx-0.35 \Omega .
$$

Both the minus sign, indicating that in the R-FoR the plume precesses in the anticyclonic direction, and the proportionality factor 0.35 agree quantitatively with our experimental measurements.

\section{Discussion}

The lateral deflection and precession were observed across all our experiments, regardless of domain size, nozzle size, buoyancy flux, and rotation rate. Preliminary experiments with rotating bubble plumes (not shown here) also exhibit plume precession. This strongly suggests that the precession is a genuine physical instability and not an artifact of our apparatus or experimental conditions.

Although none of the experimental studies mentioned in the introduction reported plume precession, experimental images from Helfrich and Battisti [1991, Figure 1c] and Goodman et al. [2004, Figure 4d] clearly show the lateral deflection of the plume. Yamamoto et al. [2011] studied the coalescing of two adjacent plumes in a rotating environment, and their experimental images (Figures $6 c-6 e$ and $8 c-8 e$ ) also show plume deflection and illustrate the "braiding" of the plumes at later times - phenomena that may be attributed to precession. The fact that Fernando et al. [1998] did not observe any precession may be due to the use of a twodimensional light sheet that obscured the three-dimensional precession, or to the running times of the experiments being too short (at our lowest rotation rate one precession period exceeds $150 \mathrm{~s}$ ). As the vertical extent $h \propto L_{B \Omega}$ of the precession region decreases with $\Omega$, this could have prevented Goodman et al. [2004] from reporting a plume precession in their experiments. Their operating conditions give $L_{B \Omega} \lesssim 2 \mathrm{~cm}$, implying that a cloud of dyed fluid may have obscured the flow up to the nozzle. The finite size of the source may also play a role if $L_{B \Omega} \sim d_{0}$.

We did not detect any dependence of the precession on Ro since $\bar{\omega} \approx 0.4 \Omega$ for all $0.02 \leq R o \leq 1.3$. We also did not find a critical Rossby number above which there was no precession. This suggests that the conventional Rossby number (the ratio of the initial risetime over a fixed height $H$ to the rotation period) cannot fully characterize the plume dynamics in a rotating environment. We believe the initial risetime ceases to be important when the plume is maintained over many rotation periods and entrains ambient fluid from ever increasing distances. Conservation of angular momentum implies that rotational effects will always become important if the plume is maintained for a sufficiently long time, provided the domain is large enough. For $\Omega \rightarrow 0$, the precession takes an infinitely long time to start. For laboratory experiments, the horizontal entrainment distance can be larger than the horizontal dimensions of the tank if $\Omega$ is small enough. However, plumes in the ocean or the atmosphere can be considered to be released into an infinite domain. Thus, we hypothesize that precession can occur for geophysical plumes after approximately $1 / \sin \theta$ days (where $\theta$ is the latitude).

A homogeneous environment was used for our study, whereas geophysical plumes in the ocean are often released in a stratified environment. However, Fabregat Tomàs et al. [2016] reported plume precession in numerical simulations of a stratified rotating environment. Another factor present in the oceans and atmosphere, but absent in our experiments, is background currents or winds. The speed of bottom oceanic currents is $\approx 1$ to $2 \mathrm{~cm} \mathrm{~s}^{-1}$ at $3000 \mathrm{~m}$ depth [Korgen et al., 1970]. Consider a plume discharged at the bottom of the ocean from a $1 \mathrm{~m}$ source. We observed a lateral deflection of up to $10 d_{0}$ at certain heights, which corresponds to $10 \mathrm{~m}$ in this case. The circumferential velocity of the plume at such height, assuming $\bar{\omega} \approx 0.4 \Omega$, would be about $0.3 \mathrm{~cm} \mathrm{~s}^{-1}$. This value is smaller than the speed of ocean currents but not negligible. The interaction between background currents and plume precession would therefore be another interesting question.

We have not found systematic observations of atmospheric or oceanic plumes over long enough periods to confirm the precession behavior. For atmospheric plumes, strong winds could disrupt the precession. For oceanic deepwater plumes, technical challenges render observations difficult. However, a possible observation of plume precession may be found in the satellite images of the plume resulting from Mount Pinatubo's volcanic eruption, 12-16 June 1991 [Holasek et al., 1996]. As the vertical component of rotation at this latitude yields a period of approximately 4 days, plume precession could have developed. On 15 June, a violent eruption rose through a previous plume up to $37 \mathrm{~km}$ height and formed a lateral intrusion. Hourly GMS visible satellite data show that the lateral intrusion remained symmetrical during the first $4-5 \mathrm{~h}$, and the plume spreading rate was significantly larger than wind speed [Holasek et al., 1996]. Then, the plume center started 
Acknowledgments This research was made possible by a grant from The Gulf of Mexico Research Initiative [10.13039/100007240] (SA 15-15), through the CARTHE consortium. Data are publicly available through the Gulf of Mexico Research Initiative Information and Data Cooperative (GRIIDC) at https://data.gulfresearchinitiative.org JRL acknowledges further support from Magdalene College, Cambridge, UK. DF acknowledges further support from Fitzwilliam College, Cambridge, UK. We would like to thank D. Page-Croft and P. Mitton for the technical support with our experimental apparatus. slowly drifting westward [Holasek et al., 1996; Chakraborty et al., 2009]. This slow lateral drift may be a hint of the onset of the plume precession. The underwater Håkon Mosby mud volcano, at $1250 \mathrm{~m}$ depth on the Norwegian-Barents-Spitzbergen margin, constantly releases gaseous methane [Sauter et al., 2006]. On 8 July 2003, Sauter et al. [2006] recorded acoustic images of the methane plume. Their Figure $3 \mathrm{c}$ shows the maximum horizontal spreading in the water column between 2:44-8:00 and 11:00-14:28. As can be clearly observed in that image, the discharge direction of the plume moves in the anticyclonic direction. At this latitude, the rotation period of the environment is about 1 day. We would therefore expect the plume to precess approximately $70^{\circ}$ in the anticyclonic direction during $12 \mathrm{~h}$ observations, which is in agreement with the acoustic measurements of the plume.

As the plume precesses and changes direction, dispersion in the environment is enhanced. As an illustration, we show in Figure 4 (right), the average normalized dye concentration of three plumes (Series $D$ ) with increasing $\Omega$ (from top to bottom). The vertical extent of the region shown corresponds to $\approx 10 d_{0}(7.5 \mathrm{~cm})$, and the plume was visibly precessing in that region for all three experiments. The yellow dashed lines indicate a theoretical plume profile in a nonrotating environment assuming $\alpha=0.1$. The width of the average dye distribution grows with increasing $\Omega$ and is broader than the nonrotating case, demonstrating that the dispersion of the plume fluid in the environment increases. Additionally, since the plume discharges at an angle to the vertical, we expect that the instantaneous highest concentrations of plume material would be found offset from the centerline. Consequently, measuring pollutant concentrations centered above the source could yield misleading results about the plume composition.

This study has revealed unique dynamics of a turbulent plume in a rotating environment, with important implications for the dispersion of pollutants in the ocean and atmosphere. We have shown that the plume axis precesses at a rate proportional to the background rotation rate and that this precession can occur no matter how small the rotation rate is. The precession leads to increased lateral dispersion of the plume and may have consequences for the eventual height of rise in a stratified environment.

\section{References}

Bush, J. W. M., and A. W. Woods (1999), Vortex generation by line plumes in a rotating stratified fluid, J. Fluid Mech., 388, 289-313, doi:10.1017/S0022112099004759.

Chakraborty, P., G. Gioia, and S. W. Kieffer (2009), Volcanic mesocyclones, Nature, 458(7237), 497-500, doi:10.1038/nature07866.

Fabregat Tomàs, A., A. C. Poje, T. M. Özgökmen, and W. K. Dewar (2016), Effects of rotation on turbulent buoyant plumes in stratified environments, J. Geophys. Res. Oceans, 121, 5397-5417, doi:10.1002/2016JC011737.

Fernando, H. J. S., R.-R. Chen, and B. A. Ayotte (1998), Development of a point plume in the presence of background rotation, Phys. Fluids, 10(9), 2369-2383, doi:10.1063/1.869754.

Goodman, J. C., G. C. Collins, J. Marshall, and R. T. Pierrhumbert (2004), Hydrothermal plume dynamics on Europa: Implications for chaos formation, J. Geophys. Res., 109, E03008, doi:10.1029/2003JE002073.

Helfrich, K. R., and T. M. Battisti (1991), Experiments on baroclinic vortex shedding from hydrothermal plumes, J. Geophys. Res., 96(C7), $12,511-12,518$, doi:10.1029/90JC02643.

Holasek, R. E., S. Self, and A. W. Woods (1996), Satellite observations and interpretation of the 1991 Mount Pinatubo eruption plumes, J. Geophys. Res., 101(B12), 27,635-27,655, doi:10.1029/96JB01179.

Huang, N. E., and N. O. Attoh-Okine (Eds.) (2005), The Hilbert-Huang Transform in Engineering, Taylor and Francis, Boca Raton, Fla, USA

Korgen, B. J., G. Bodvarsson, and L. Kulm (1970), Current speeds near the ocean floor west of Oregon, Deep Sea Res. Oceanogr. Abstr., 17(2), 353-357, doi:10.1016/0011-7471(70)90026-4.

Sauter, E. J., S. I. Muyakshin, J.-L. Charlou, M. Schlüter, A. Boetius, K. Jerosch, E. Damm, J.-P. Foucher, and M. Klages (2006), Methane discharge from a deep-sea submarine mud volcano into the upper water column by gas hydrate-coated methane bubbles, Earth Planet. Sci. Lett., 243(3), 354-365, doi:10.1016/j.epsl.2006.01.041.

Socolofsky, S. A., E. E. Adams, and C. R. Sherwood (2011), Formation dynamics of subsurface hydrocarbon intrusions following the Deepwater Horizon blowout, Geophys. Res. Lett., 38, L09602, doi:10.1029/2011GL047174.

Yamamoto, H., C. Cenedese, and C. P. Caulfield (2011), Laboratory experiments on two coalescing axisymmetric turbulent plumes in a rotating fluid, Phys. Fluids, 23(5), 056601, doi:10.1063/1.3584134. 